\section{Unified analysis of channel capacity under different adaptive transmission policies}

\section{H. Al-Hmood and H. S. Al-Raweshidy}

In this letter, the channel capacity under different transmission policies is analysed using a mixture gamma (MG) distribution. Specifically, the capacity of the channel with inversion and fixed rate (CIFR), optimum power and rate adaptation (OPRA), and truncated inversion and fixed rate (TIFR) are derived in unified exact simple closed-form expressions. The composite $\alpha-\lambda-\eta-\mu /$ gamma fading channel which includes most of the well-known fading distributions as special cases, has been employed in this work. Moreover, a comparison between numerical and simulation results has been carried out to verify the validation of the derived expressions.

Introduction: The analysis of the channel capacity under different transmission policies has been given a special attention in the technical literature. For instance, the capacity of the channel with inversion and fixed rate (CIFR), optimum power rate and adaptation (OPRA), and truncated inversion and fixed rate (TIFR) over Rayleigh and Nakagami- $m$ fading channels are analysed in [1] and [2], respectively.

Recently, several works have been dedicated to analyse the capacity with adaptive transmission protocols over generalised fading channels. For example, the analysis in [3] and [4] are carried out over $\eta-\mu$ and $\alpha-\mu$ fading channels, respectively. The former model is employed to represent the non-line-of-sight (NLoS) communication scenarios whereas the latter is utilised for the non-linear environment. In [5], the capacity of composite $\eta-\mu /$ gamma fading channel is investigated. The Fox's $H$-function is used in [6] to derive unified expressions of the channel capacity for a variety of fading channels under different transmission strategies. However, there are some distributions that can not be modelled by this function such as $\alpha-\mu /$ gamma. Moreover, the Fox's $H$-function is defined in the form of Barnes-type contour integral (please see [6, eq. (2)]). Therefore, it does not provide clear insight into the impact of the fading parameters on the system performance.

Motivated by there is no general unified framework for the channel capacity under different transmission policies, this letter is devoted to fill this gap by using a mixture gamma (MG) distribution [7]. This distribution has been widely utilised in the open literature for various applications of communication systems [8,9]. This is because it gives simple closed-form expression of the performance metrics with good matching to the practical measurements. To this end, the channel capacity over composite $\alpha-\lambda-\eta-\mu$ fading condition which is proposed by [10] as a generalised model of $\alpha-\mu, \lambda-\mu$, and $\eta-\mu$, fading channel and gamma distribution is analysed.

$M G$ channel model: The probability density function (PDF) of the instantaneous signal-to-noise (SNR) using a MG distribution is expressed as [7, eq. (1)]

$$
f_{\gamma}(\gamma)=\sum_{j=1}^{M} \Xi_{j} \gamma^{\varphi_{j}-1} e^{-\rho_{j} \gamma}
$$

where $M$ is the efficient number of Gamma distributions that provides the minimum mean square error (MSE) between the approximate and exact PDFs and $\Xi_{j}, \varphi_{j}$, and $\rho_{j}$ represent the parameters of $j$ th Gamma component.

Channel capacity with OPRA: The normalised channel capacity with OPRA is given as [1, eq. (7)]

$$
C_{\text {OPRA }}=\int_{\gamma_{0}}^{\infty} \log _{2}\left(\frac{\gamma}{\gamma_{0}}\right) f_{\gamma}(\gamma) d \gamma
$$

where $\gamma_{0}$ is a threshold value that should satisfy the following identity

$$
\int_{\gamma_{0}}^{\infty}\left(\frac{1}{\gamma_{0}}-\frac{1}{\gamma}\right) f_{\gamma}(\gamma) d \gamma=1
$$

Inserting (1) in (2), this yields

$$
C_{\mathrm{OPRA}}=\sum_{j=1}^{M} \Xi_{j} \int_{\gamma_{0}}^{\infty} \gamma^{\varphi_{j}-1} e^{-\rho_{j} \gamma} \log _{2}\left(\frac{\gamma}{\gamma_{0}}\right) d \gamma
$$

Using the substitution $\gamma=\gamma_{0} t$, (4) becomes

$$
C_{\text {OPRA }}=\frac{1}{\ln 2} \sum_{j=1}^{M} \Xi_{j} \gamma_{0}^{\varphi_{j}} \int_{1}^{\infty} t^{\varphi_{j}-1} e^{-\rho_{j} \gamma_{0} t} \ln (t) d t
$$

With the aid of [11, eq. (4.358.1)], the integration of (5) can be computed in exact closed-form expression as

$$
C_{\mathrm{OPRA}}=\frac{1}{\ln 2} \sum_{j=1}^{M} \Xi_{j} \gamma_{0}^{\varphi_{j}} \frac{\partial\left\{\left(\rho_{j} \gamma_{0}\right)^{-\varphi_{j}} \Gamma\left(\varphi_{j}, \rho_{j} \gamma_{0}\right)\right\}}{\partial\left\{\varphi_{j}\right\}}
$$

where $\frac{\partial\{y(x)\}}{\partial x}$ is the partial derivative and $\Gamma(a, b)=\int_{b}^{\infty} x^{a-1 e^{-x}} d x$ is the upper incomplete gamma function [11, eq. (8.350.2)].

Invoking the identity [12, eq. $(06.06 .20 .0011 .01)]$ to evaluate the partial derivative of (6) with respect to $\varphi_{j}$. Consequently, we have

$$
C_{\text {OPRA }}=\frac{1}{\ln 2} \sum_{j=1}^{M} \frac{\Xi_{j}}{\rho_{j}^{\varphi_{j}}} G_{2,3}^{3,0}\left[\begin{array}{c}
1,1 \\
0,0, \varphi_{j}
\end{array} \mid \rho_{j} \gamma_{0}\right]
$$

where $G_{c, d}^{a, b}[$.$] is the Meijer's G$-function defined in [11, eq. (9.301)].

When $\varphi_{j}$ is an integer number for each $j$ th, (5) can be calculated by using [1, Appendix A] as follows

$$
C_{\mathrm{OPRA}}=\frac{1}{\ln 2} \sum_{j=1}^{M} \frac{\Xi_{j}}{\rho_{j}^{\varphi_{j}}} \Gamma\left(\varphi_{j}\right) \sum_{l=0}^{\varphi_{j}-1} \frac{\Gamma\left(l, \rho_{j} \gamma_{0}\right)}{l !}
$$

where $\Gamma(a)=\Gamma(a, 0)$ is the incomplete gamma function.

Capacity with CIFR: The normalised capacity with CIFR can be evaluated by [1, eq. (46)]

$$
C_{\mathrm{CIFR}}=\log _{2}\left(1+\left(\int_{0}^{\infty} \frac{f_{\gamma}(\gamma)}{\gamma} d \gamma\right)^{-1}\right)
$$

Plugging (1) in (9) and employing [11, eq. (3.381.4)] to calculate the integral. Thus, this yields

$$
C_{\mathrm{CIFR}}=\log _{2}\left(1+\left(\sum_{j=1}^{M} \frac{\Xi_{j}}{\rho_{j}^{\varphi_{j}-1}} \Gamma\left(\varphi_{j}-1\right)\right)^{-1}\right)
$$

One can see that (9) is valid for $\varphi_{j} \geqslant 1$

Capacity with TIFR: The normalised capacity with TIFR is evaluated by [1, eq. (47)]

$$
C_{\mathrm{TIFR}}=\log _{2}\left(1+\left(\int_{\gamma_{0}}^{\infty} \frac{f_{\gamma}(\gamma)}{\gamma} d \gamma\right)^{-1}\right)\left(1-F_{\gamma}\left(\gamma_{0}\right)\right)
$$

where $\gamma_{0}$ is the predefined threshold value and $F_{\gamma}\left(\gamma_{0}\right)$ stands for the cumulative distribution function (CDF) of the instantaneous SNR at $\gamma_{0}$ that is given in [7, eq. (2)].

Substituting (1) into (11) and recalling [11, eq. (3.381.3)] to compute the integral, the result is

$$
C_{\mathrm{TIFR}}=\log _{2}\left(1+\left(\sum_{j=1}^{M} \frac{\Xi_{j}}{\rho_{j}^{\varphi_{j}-1}} \Gamma\left(\varphi_{j}-1, \rho_{j} \gamma_{0}\right)\right)^{-1}\right)\left(1-F_{\gamma}\left(\gamma_{0}\right)\right)
$$

Modelling of composite $\alpha-\lambda-\eta-\mu /$ gamma fading channel using a $M G$ distribution: The PDF of the instantaneous SNR over composite $\alpha-\lambda-\eta-\mu /$ gamma fading can be obtained by averaging the PDF of $\alpha-\lambda-\eta-\mu$ [10, eq. (10)] over gamma distribution [5, eq. (5)]. Accordingly, this yields

$$
\begin{aligned}
f_{\gamma}(\gamma) & =\frac{\sqrt{\pi} \alpha\left(\mu\left(1+\eta^{-1}\right)\right)^{\mu+\frac{1}{2}}}{\Gamma(\mu) \Gamma(k) \Omega^{k} b^{\mu-\frac{1}{2}}}\left(\frac{\eta}{1-\lambda^{2}}\right)^{\mu} \gamma^{\alpha\left(\mu+\frac{1}{2}\right)-1} \\
& \times \int_{0}^{\infty} y^{k-\alpha\left(\mu+\frac{1}{2}\right)-1} e^{-c \frac{\gamma^{\alpha}}{y^{\alpha}}-\frac{y}{\Omega}} I_{\mu-\frac{1}{2}}\left(\frac{\mu(1+\eta) b \gamma^{\alpha}}{2 \eta y^{\alpha}}\right) d y
\end{aligned}
$$

where $\alpha, \mu, k$, and $\Omega$ are the non-linearity severe index, the real extension of the number of multipath clusters, shadowing severity parameter, and mean power, respectively. Moreover, $I_{a}($.$) is the modified Bessel$ 
function of the first kind and $a$ th order [11, eq. (8.440)]. The parameter $\eta$ denotes the power ratio between the quadrature and in-phase scattered components whereas $\lambda$ stands for the correlation coefficient between them. The parameters $b$ and $c$ are given as

$$
b=\frac{\sqrt{(\eta-1)^{2}+4 \eta \lambda^{2}}}{1-\lambda^{2}} \quad c=\frac{\mu(1+\eta)^{2}}{2 \eta\left(1-\lambda^{2}\right)}
$$

Using the substitution $x=c \frac{\gamma^{\alpha}}{y^{\alpha}}$ into (13), we have

$$
\begin{aligned}
f_{\gamma}(\gamma) & =\frac{\sqrt{\pi}\left(\mu\left(1+\eta^{-1}\right)\right)^{\mu+\frac{1}{2}} c^{\frac{k}{\alpha}-\mu-\frac{1}{2}}}{\Gamma(\mu) \Gamma(k) \Omega^{k} b^{\mu-\frac{1}{2}}}\left(\frac{\eta}{1-\lambda^{2}}\right)^{\mu} \gamma^{k-1} \\
& \times \int_{0}^{\infty} x^{\mu-\frac{k}{\alpha}-\frac{1}{2}} e^{-\left(\frac{c}{x}\right)^{\frac{1}{\alpha}} \frac{\gamma}{\Omega}-x} I_{\mu-\frac{1}{2}}\left(\frac{\mu(1+\eta) b}{2 \eta c} x\right) d x
\end{aligned}
$$

It can be noticed that (15) can be expressed as

$$
f_{\gamma}(\gamma)=\frac{\sqrt{\pi}\left(\mu\left(1+\eta^{-1}\right)\right)^{\mu+\frac{1}{2}} c^{\frac{k}{\alpha}-\mu-\frac{1}{2}} \eta^{\mu} \gamma^{k-1}}{\Gamma(\mu) \Gamma(k) \Omega^{k} b^{\mu-\frac{1}{2}}\left(1-\lambda^{2}\right)^{\mu}} \int_{0}^{\infty} e^{-x} z(x) d x
$$

where $z(x)=x^{\mu-\frac{k}{\alpha}-\frac{1}{2}} e^{-\left(\frac{c}{x}\right)^{\frac{1}{\alpha}} \frac{\gamma}{\Omega}} I_{\mu-\frac{1}{2}}\left(\frac{\mu(1+\eta) b}{2 \eta c} x\right)$.

The integration in (16), $\mathcal{P}=\int_{0}^{\infty} e^{-x} z(x) d x$ can be highly approximated using a Gaussian-Laguerre quadrature sum, $\mathcal{P}=$ $\sum_{j=1}^{M} w_{j} z\left(x_{j}\right)$ where $w_{j}$ and $x_{j}$ are the abscissas and weight factors provided in [13]. Hence, (16) can be rewritten using a MG distribution with the equivalent parameters

$$
\begin{array}{r}
\Xi_{j}=\frac{\theta_{j}}{\sum_{i=1}^{M} \theta_{i} \Gamma\left(\varphi_{i}\right) \rho_{i}-\varphi_{i}}, \quad \varphi_{j}=k, \quad \rho_{j}=\frac{c^{\frac{1}{\alpha}}}{\Omega x_{j}^{\frac{1}{\alpha}}}, \\
\theta_{j}=\frac{\sqrt{\pi}\left(\mu\left(1+\eta^{-1}\right)\right)^{\mu+\frac{1}{2}} c^{\frac{k}{\alpha}-\mu-\frac{1}{2}} \eta^{\mu}}{\Gamma(\mu) \Gamma(k) \Omega^{k} b^{\mu-\frac{1}{2}}\left(1-\lambda^{2}\right)^{\mu}} w_{j} x_{j}^{\mu-\frac{k}{\alpha}-\frac{1}{2}} \\
\times I_{\mu-\frac{1}{2}}\left(\frac{\mu(1+\eta) b}{2 \eta c} x_{j}\right)
\end{array}
$$

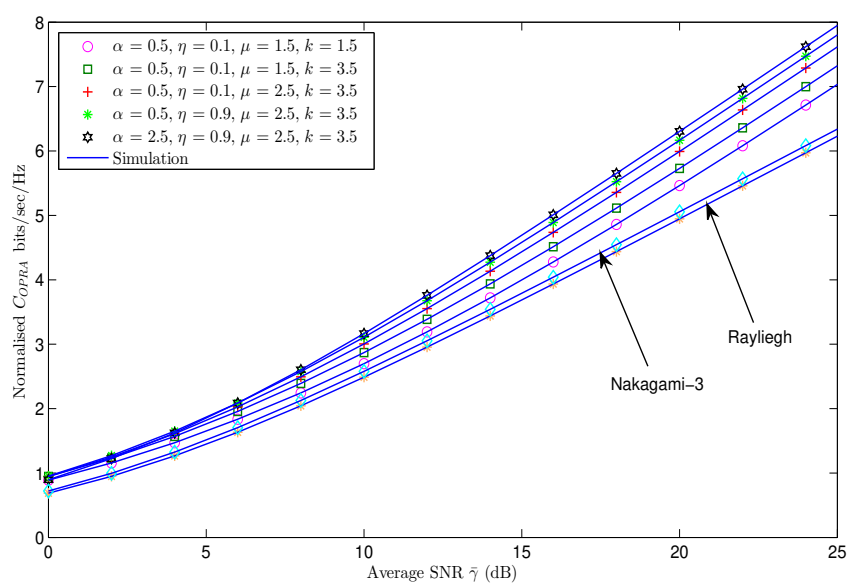

Fig. 1 Normalised capacity with OPRA against average SNR, $\bar{\gamma}$, over $\alpha-$ $\lambda-\eta-\mu /$ gamma fading for different values of $\alpha, \eta, \mu, k$ and $\lambda=0.1$

Numerical results: Fig. 1 and Fig. 2 demonstrate the normalised capacity with OPRA and CIFR and TIFR over composite $\alpha-\lambda-\eta-\mu /$ gamma fading channel against the average SNR, $\bar{\gamma}$, respectively, for different values of the fading parameters. The number of components, namely, $M$, is chosen to achieve MSE $\leq 10^{-8}$. From both figures, it can be observed that the channel capacity improves when $\alpha, \eta, \mu$ or/and $k$ increase. This is because higher $\alpha, \eta, \mu$ and $k$ mean the non-linearity effect of the channel is low, the number of multipath clusters is large, the received power is high and the shadowing impact is reduced, respectively. In addition, by inserting $\alpha=2, \lambda \rightarrow 0, \eta=1, k \rightarrow \infty$ and $\mu=1$ for Rayleigh and $\mu=3$ for Nakagami-3 in eq. (17), the $C_{\text {OPRA }}$ for the aforementioned fading channels can be deduced from eq. (7) as shown in Fig. 1.

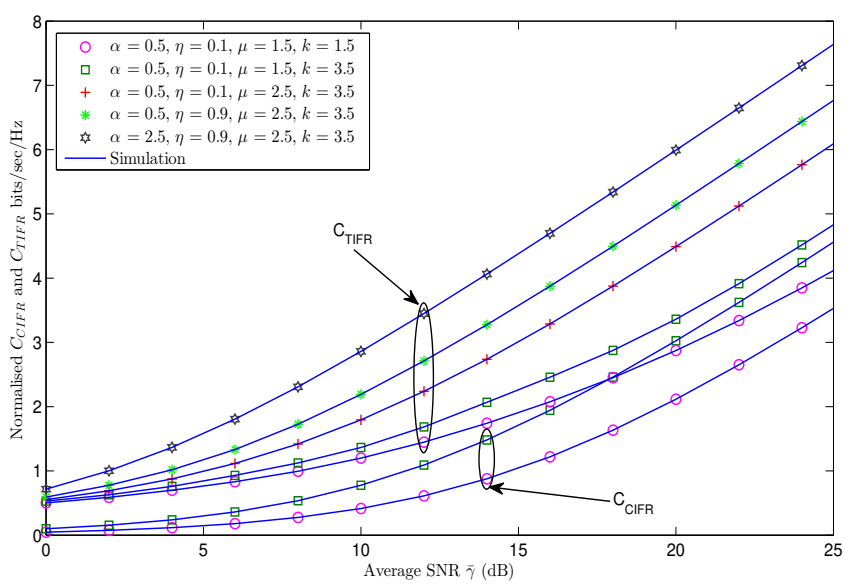

Fig. 2 Normalised capacity with CIFR and TIFR against average SNR, $\bar{\gamma}$, over $\alpha-\lambda-\eta-\mu /$ gamma fading for different values of $\alpha, \eta, \mu, k$ and $\lambda=0.1$

Conclusion: In this letter, a MG distribution was used to derive a unified framework of the channel capacity under different transmission policies. The PDF of a MG distribution is expressed in terms of the power and exponential functions. To this effect, simple closed-form mathematically tractable expressions of the capacity with OPRA, CIFR, and TIFR were obtained. The derived results were applied for the composite $\alpha-\lambda-$ $\eta-\mu /$ gamma fading channels that have not been yet investigated in the technical literature. Furthermore, the results of this work can be employed for all the special cases of $\alpha-\lambda-\eta-\mu /$ gamma fading which are most of the conventional, generalised and composite distributions such as Nakagami- $m$, and $\alpha-\eta-\mu /$ gamma.

(c)

Hussien Al-Hmood (Electronic and Computer Engineering (ECE) Department, College of Engineering, Design and Physical Sciences, Brunel University London, UK)

E-mail: hussien.al-hmood@brunel.ac.uk, eng.utq.edu.iq

H. S. Al-Raweshidy (ECE Department, College of Engineering, Design and Physical Sciences, Brunel University London, UK)

\section{References}

1 Alouini, M.-S. and Goldsmith, A.J.: 'Capacity of Rayleigh fading channels under different adaptive transmission and diversity-combining techniques,' IEEE Trans. Veh. Technol., 1999, 48, (4), pp. 1165-1181

2 Alouini, M.-S. and Goldsmith, A.J.: 'Adaptive modulation over Nakagami fading channels,' Wireless Pers. Commun., 2000, 13, (1-2), pp. 119-143

3 Peppas, K.P.: 'Capacity of $\eta-\mu$ fading channels under different adaptive transmission techniques,' IET Commun., 2010, 4, (5), pp. 532-539

4 Mohamed, R., et al.: 'Closed-form capacity expressions for the $\alpha-\mu$ fading channel with SC diversity under different adaptive transmission strategies,' Frequenz, 2013. 67, (3-4), pp. 127-137

5 Zhang, J., Matthaiou, M., Tan, Z., and Wang, H.: 'Performance analysis of digital communication systems over composite $\eta-\mu$ /gamma fading channels,' IEEE Trans. Veh. Technol., 2012, 61, (7), pp. 3114-3124

6 Rahama, Y.A., et al.: 'Capacity of Fox's $H$-function fading channel with adaptive transmission', Electron. Lett., 2016, 52, (11), pp. 976-978

7 Atapattu, S., Tellambura, C., and Jiang, H.: 'A mixture gamma distribution to model the SNR of wireless channels', IEEE Trans. Wirel. Commun., 2011, 10, (12), pp. 4193-4203

8 Al-Hmood, H., and Al-Raweshidy, H.S.: 'Unified modeling of composite $\kappa-\mu /$ gamma, $\eta-\mu /$ gamma, and $\alpha-\mu /$ gamma fading channels using a mixture gamma distribution with applications to energy detection,' IEEE Antennas Wirel. Propag. Lett., 2017, 17, pp. 104-108

9 Al-Hmood, H., and Al-Raweshidy, H.S.: 'Unified approaches based effective capacity analysis over composite $\alpha-\eta-\mu /$ gamma fading channels', Electron. Lett., 2018, 54, (13), pp. 852-853

10 Papazafeiropoulos, A.K., Kotsopoulos S.A., and Zevgolis, D.: 'The $\alpha-$ $\lambda-\eta-\mu$ : A general fading distribution', Proc. IEEE Wirel. Commun. and Net. Conf. (WCNC), Budapest, 2009, pp. 1-6

11 Gradshteyn, I.S. and Ryzhik, I.M.: 'Table of integrals, series, and products', (7th ed. San Diego, CA: Academic Press, 2007)

12 Wolfram, The Wolfram functions site. Available: http://functions.wolfram.com, 2019

13 Abramowitz, M., and Stegun, I.A.: 'Handbook of mathematical functions with formulas, graphs, and mathematical tables', (9th edition, Dover, 1970) 